\title{
Age-Related Neural Dedifferentiation and Cognition
}

Joshua D. Koen ${ }^{a^{*}}$, Sabina Srokova ${ }^{b}$, and Michael D. Rugg ${ }^{\mathrm{b}}$

${ }^{\mathrm{a} D e p a r t m e n t}$ of Psychology, University of Notre Dame, 390 Corbett Family Hall, Notre Dame, IN, 46556

${ }^{b}$ Center for Vital Longevity and School of Behavioral and Brain Sciences, University of Texas at Dallas, 1600 Viceroy Dr., Suite 800, Dallas, TX, 75235 


\begin{abstract}
This review focuses on possible contributions of neural dedifferentiation to age-related cognitive decline. Neural dedifferentiation is held to reflect a breakdown in the functional specificity of brain regions and networks that compromises the fidelity of neural representations supporting episodic memory and related cognitive functions. The evidence for age-related dedifferentiation is robust when it is operationalized as neural selectivity for different categories of perceptual stimuli or as decreased segregation or modularity of resting-state functional brain networks. Neural dedifferentiation for perceptual categories appears to demonstrate a negative, age-invariant relationship with performance on tests of memory and fluid processing. Whether this pattern extends to network-level measures of dedifferentiation cannot currently be determined due to insufficient evidence. The existing data highlight the importance of further examination of neural dedifferentiation as a factor contributing to episodic memory and to cognitive performance more generally.
\end{abstract}




\section{Introduction}

Many cognitive abilities decline in the course of healthy aging, including episodic memory, executive control and processing speed [1]. Identifying the factors that cause and moderate cognitive aging is a pressing concern given the rapid rate at which the population is aging [2]. One factor that has received increased interest recently is neural dedifferentiation. This term refers to findings suggesting that neural processing becomes less selective with increasing age. Neural dedifferentiation is hypothesized to compromise the fidelity and efficiency of the neural representations and processes that support episodic memory and other aspects of cognition $[3,4]$.

The concept of dedifferentiation originated from behavioral findings reporting an age-related increase in correlations between different cognitive and sensory abilities [5] (recently termed 'static' cognitive dedifferentiation; see [6••]). Although these findings have not held up to recent scrutiny (cf. [7]), they motivated early investigations aimed at identifying a neural counterpart $[3,4]$.

We provide an update on studies that examined age-related neural dedifferentiation with fMRI (for more extensive treatment of some of the topics discussed below, see [7]). First, we provide a brief review of research in non-human animals that suggests a mechanistic basis for neural dedifferentiation. Then, we provide a selective review of fMRI studies examining dedifferentiation at three different levels of investigation (individual items, perceptual categories, and functional networks - a level that was not covered previously [7]). Finally, we review findings examining relationships between different indices of neural differentiation and cognitive performance, with an emphasis on dedifferentiation of functional networks.

\section{Age-related dedifferentiation in non-human animals}

Animal research has operationalized neural dedifferentiation in terms of the broadening of receptive fields of single neurons. Initial studies $[8,9]$ reported reduced orientation and directional selectivity of neurons in striate cortex (V1) of senescent macaques. Similar findings have been reported in other feature-selective cortical regions and stimulus modalities (e.g., [10-12]) and in other non-human animal species $[13,14]$. It remains to be seen whether these findings extend to neural populations, however. Of importance, this literature has identified age-related reductions in gamma-aminobutyric acid (GABA) inhibitory neurotransmission $[9,14]$ as a potential mechanism for neural dedifferentiation. Building on these findings, recent studies in humans have provided suggestive evidence that reductions in the concentration of cortical GABA are associated with age-related neural dedifferentiation at both the level of perceptual categories [15] and large-scale brain networks [16].

\section{Item-Level Neural Dedifferentiation}

Staying with research on humans, several studies have examined age-related neural dedifferentiation for individual items (Figure 1). One approach is to quantify neural pattern similarity between successive repetitions of the same item relative to different items, under the assumption that reduced pattern similarity reflects neural dedifferentiation (see also [17]). One study reported null effects of age for repetitions of brief movie clips during a memory encoding task [18,19]. Another study [20], contrasting face, scene, and object stimuli, also reported a null item-level effect of age (after controlling for age differences in baseline similarity scores; cf. Figure 1A). In contrast, a third study [21] reported agerelated reductions in measures of neural differentiation for individual scene and object exemplars. 
However, this conclusion was based on the finding that measures of item-level similarity were significantly above baseline in young but not older adults, rather than on a direct comparison between the two age groups.

Another approach to examining item-level neural differentiation is to exploit the phenomenon of fMRI adaptation or 'repetition suppression'. This takes the form of a reduction in the neural response elicited by an item when it is repeated and is thought to be a consequence of re-accessing the same neural representation [22]. Studies using this approach have compared the neural responses elicited by item repetitions to the responses elicited by items that are highly similar, but not identical, to a previously presented exemplar. The assumption is that suppression effects for similar exemplars are indicative of item-level neural dedifferentiation (Figure 1B). The first study adopting this approach [23] reported equivalent repetition suppression in the 'fusiform face area' (see below) of young and older adults for exact repetitions of faces, but greater suppression in older adults for faces that were visually similar to first-presented faces. Analogous findings have been reported for object stimuli in the entorhinal cortex [24] and hippocampus [25].

A recent study [26••] trained multivariate classifiers to discriminate between re-presentations of studied object images (targets) and two classes of related 'lure' objects - visually similar 'item lures', and visually dissimilar, but conceptually related 'thematic lures' - during a recognition memory test. Separate classifiers were trained to discriminate between targets and each class of lure. Among a priori defined regions-of-interest, age effects were confined to middle occipital cortex, where classification accuracy of targets and item lures was above chance for young, but not older adults. This finding is suggestive of low precision target representations (i.e., item-level dedifferentiation) in older adults.

The findings from the above studies lend support to the proposal [3] that age-related dedifferentiation can be identified at the level of individual items. The boundary conditions of the phenomenon are unclear however, as is the question of whether it extends to stimulus categories other than faces and objects.

\section{Category-Level Neural Dedifferentiation}

Category-level dedifferentiation has mainly been operationalized as a reduction in the selectivity of fMRI BOLD activity elicited by different visual categories (e.g., images of scenes, objects, or faces; see Figure 2) $[7,27,28,29 \bullet]$. These studies are rooted in the large literature documenting category-selective neural responses in different regions of ventral occipital-temporal cortex [30]. Importantly, analogous findings have been reported in both motor [31] and auditory [15,32] cortical systems, suggesting that agerelated neural dedifferentiation at this level extends beyond the visual domain.

In a pioneering study, Park and colleagues [27] reported the first evidence for category-level age-related neural dedifferentiation. They examined the selectivity of BOLD responses elicited by houses, objects, faces, and pseudowords and described an age-related reduction in the selectivity of the responses for each stimulus category. Research over the past 15 years has largely replicated these findings. The most consistent evidence for category-level dedifferentiation has come from studies examining neural selectivity for images of faces $[27,28,33]$ and scenes $[20,27,28,29 \bullet]$, in the fusiform face area (FFA) and parahippocampal place area (PPA), respectively. However, two notable exceptions reported null age differences for face stimuli in the FFA [34] and scene stimuli in the PPA [35]. 
In contrast to faces and scenes, evidence of age-related dedifferentiation for other visual categories is equivocal. Studies employing visual words and images of objects have mainly reported null effects of age on neural selectivity in, respectively, word-selective $[28,36,37]$ and object-selective $[20,29 \bullet, 38]$ cortical regions. Null effects of age were also reported for color patches in color-selective extrastriate visual cortex [28]. One recent study did however report evidence of age-related dedifferentiation for objects in perirhinal cortex [35], a region that is also implicated in object processing (e.g., [39]).

Numerous factors likely contribute to these inconsistent findings. One potentially important factor is across-study differences in task demands (e.g., passive versus active viewing of the stimuli). For instance, age-related neural dedifferentiation has been reported for both faces $[27,28]$ and visual objects [27] when participants passively view the images (but see [38]). However, two studies that employed tasks requiring a discriminative judgment to each item reported no evidence for age-related neural dedifferentiation for faces [29] or objects [20•] (see also [30]). Another important factor might be the nature of the experimental stimuli. Studies using visual objects have drawn stimuli from a single [18] versus multiple $[6,33]$ sub-categories, with age-related dedifferentiation only observed in the former case (and during passive viewing). Lastly, age differences in cumulative life experience with exemplars belonging to different perceptual categories may make a critical contribution to age differences in category-level measures of neural differentiation [7].

\section{Dedifferentiation at the Network Level}

Spontaneous ('resting state') brain activity measured with fMRI demonstrates systematic patterns of inter-regional correlations that can be decomposed into highly reproducible large-scale brain networks (sometimes referred to as 'systems', 'communities', or 'sub-networks'; for review, see [40]). The application of graph theoretic analyses to these patterns of resting state connectivity enables quantification of the extent to which the brain (characterized as a distributed set of 'nodes') can be segregated into functional networks (Figure 3). A segregated brain is defined by dense connectivity among nodes in the same network (i.e., high within-network connectivity) coupled with sparse connections between nodes belonging to different networks (i.e., low between-network connectivity). Network-level neural differentiation can be operationalized in terms of the ratio of within- to betweennetwork connectivity or the related metric of 'modularity'. A number of studies have examined the effects of age on resting state connectivity data by employing these or similar metrics (see [41] for review). Overall, the findings have consistently demonstrated a trend toward decreased segregation or modularity with increasing age.

For example, Chan and colleagues [42] examined the effects of age on network segregation in a large lifespan sample (range 20-89 years) of cognitively healthy adults (for similar findings, see [43]). The authors reported that segregation declined linearly with increasing age in 'sensorimotor' networks but with a positively accelerating trend in 'associative' networks (i.e., networks implicated in higher-level cognitive functions; Figure 3 ). The same group recently reported closely similar findings [44•] using agespecific node sets (albeit now identifying a cubic relationship between age and segregation of associative networks). Importantly, the data from this more recent study suggest that prior results are not a consequence of age-related bias in node selection, which is an important precursor to networklevel analysis [40]. Two other recent studies that adopted a lifespan approach reported similar findings. One study [45] reported that network modularity declined linearly with age in a sample of participants aged 7 to 85 years (also see $[43,46,47]$ ). In another study [48] within-network connectivity was 
unaffected by age whereas between-network connectivity linearly increased, a pattern indicative of agerelated network desegregation. Other recent findings adopting extreme age-group designs have reported age-related desegregation of networks in three sensorimotor and two associative networks [16], and age-related decreases in network modularity collapsed across networks [49,50,51••]. The study by Chong and colleagues [ $51 \bullet \bullet$ ] is especially notable because it reported longitudinal evidence (across intervals ranging from 18 months to 5 years) of within-individual declines in network modularity in a sample of older adults.

The above findings provide strong evidence that the large-scale functional networks identified in restingstate $\mathrm{fMRI}$ data show evidence of age-related neural dedifferentiation. It remains an open question whether network-level dedifferentiation varies in magnitude between different classes of networks (e.g., sensorimotor vs. associative). Moreover, it is unclear if analogous effects will emerge when taskrelated patterns of inter-regional connectivity are subjected to similar analytic methods (cf. $[48,50]$ ). It is also unclear whether and how network desegregation might be associated with item-level and categorylevel dedifferentiation (cf. [52・•]).

\section{Relationships with Cognitive Performance}

Neural dedifferentiation has been proposed as an important factor contributing to age-related cognitive decline $[3,4]$ (see [7] for a recent review). This may be particularly important for explaining age-related declines in episodic memory because the encoding and retrieval processes supporting episodic memory depend upon high fidelity neural representations $[25,29,26,18,19]$. The foregoing proposal is supported by evidence that neural dedifferentiation at both the item $[23,25,26]$ and category levels $[29 \bullet, 32,35,53]$ is associated with poorer performance on tasks that engage 'fluid' processing, including episodic memory $[25,26,29,35]$. Importantly, the evidence suggests that these relationships do not differ in strength as a function of age (i.e., they are age-invariant). While findings of age-invariance are consistent with proposals that item- and category-level dedifferentiation mediate age-related cognitive decline, they also suggest that high neural selectivity confers similar benefits to cognition across much of the adult lifespan (cf. [54]).

Only a few studies have examined relationships between network-level dedifferentiation and cognitive performance. One study [42] reported a positive relationship between episodic memory performance (as assessed with a standardized test battery) and segregation of associative, but not sensorimotor, networks. Using a similar approach, a recent study [43] reported a positive relationship between wholebrain system segregation (but not modularity) and episodic memory, processing speed, and fluid intelligence constructs. While the brain-behavior relationship remained significant after controlling for age in one of the above studies [42], neither reported whether age moderated the strength of the relationship. Another recent experiment [49] reported that relatively greater network segregation (indexed by three different metrics) correlated positively with training-related gains in working memory capacity in older adults, while the analogous correlations in young participants were not reported. A complex pattern of brain-behavior relationships that defy simple summary was reported in another recent study [50]. As the authors of the study noted, however, none of the reported correlations survived correction for multiple comparisons and thus should be treated as suggestive only.

Two other studies examined relationships between metrics related to network segregation and performance on tests of motor function. In one study [48] increased between-network connectivity was associated with poorer accuracy on a bimanual coordination task in a cross-sectional lifespan sample of 
healthy adults. This relationship persisted after controlling for within-network connectivity in 14 different functional networks, including motor networks. The relationship was not examined after controlling for the effects of age, although age was positively correlated with between-network connectivity and negatively correlated with motor performance. In another recent study [16] desegregation of sensorimotor networks was associated with poorer sensorimotor performance in an age-invariant manner.

It is noteworthy that only one of the above studies [16] directly examined whether there was a moderating influence of age on relationships between measures of dedifferentiation and behavior. Thus, it remains an open question as to whether measures of network-level dedifferentiation converge with item-level and category-level measures in demonstrating age-invariant relationships with cognitive performance [7].

\section{Concluding Remarks}

There is robust evidence in humans for age-related neural dedifferentiation at the item-, category-, and network-levels of analysis. It is possible that these findings, which rely exclusively on $\mathrm{fMRI}$, might be confounded by age differences in neurovascular function [55]. We note however that several of the measures used in the studies reviewed here are insensitive to age differences in neurovascular coupling [7]. Moreover, it is difficult to reconcile findings that neural dedifferentiation can be material-, regionand network-specific with a vascular account.

There is also strong evidence that neural dedifferentiation correlates negatively with cognitive performance, including performance on tests of episodic memory. No studies to our knowledge have examined relationships between neural differentiation and other domains of memory, such as semantic or procedural memory. Importantly, the available evidence suggests that these brain-behavior relationships are largely age-invariant, at least for item- and category-level measures. Such findings suggest that these relationships likely reflect general principles of neural function and organization that operate across the adult lifespan.

Only one study [51 ••] has reported age-related dedifferentiation using a longitudinal rather than a cross-sectional design. Therefore, it remains to be firmly established that the phenomenon, especially when operationalized using task-based measures, reflects aging rather than one or more confounding factors (e.g. cohort effects, cf. [54]). Additional research is also needed to determine if neural dedifferentiation at item and category levels extends beyond perceptual stimuli [56-59]. Finally, future research would benefit by including other neuroimaging measures, such as EEG/MEG, to provide additional insights into the functional significance and mechanisms of age-related neural dedifferentiation. 


\section{Acknowledgements}

This work was supported by the National Institutes of Health [grant number AG039103] and the National Science Foundation [grant number 1633873]. The authors thank Micaela Chan and Gagan Wig for providing the images for Figure 3. 


\section{References}

1. Salthouse TA: Trajectories of normal cognitive aging. Psychol Aging 2019, 34:17-24.

2. United Nations, Department of Economic and Social Affairs, Population Division: World Population Prospects: The 2017 Revision, Key Findings and Advance Tables. United Nations; 2017.

3. Li S-C, Lindenberger U, Sikström S: Aging cognition: from neuromodulation to representation. Trends Cogn Sci 2001, 5:479-486.

4. Li S-C, Rieckmann A: Neuromodulation and aging: implications of aging neuronal gain control on cognition. Curr Opin Neurobiol 2014, 29:148-158.

5. Baltes PB, Lindenberger U: Emergence of a Powerful Connection Between Sensory and Cognitive Functions Across the Adult Life Span: A New Window to the Study of Cognitive Aging? Psychol Aging 1997, 12:12-21.

6. $\bullet$ Tucker-Drob EM, Brandmaier AM, Lindenberger U: Coupled cognitive changes in adulthood: A meta-analysis. Psychol Bull 2019, 145:273-301. Meta-analytic review of longitudinal studies of age-changes in correlations between different cognitive abilities. Reported little or no evidence for age-related increases in the correlation between abilities in different cognitive domains ('static dedifferentiation'), but strong evidence of age-related increases in coupling of change scores across domains (dynamic dedifferentiation).

7. Koen JD, Rugg MD: Neural Dedifferentiation in the Aging Brain. Trends Cogn Sci 2019, 23:547-559.

8. Schmolesky MT, Wang Y, Pu M, Leventhal AG: Degradation of stimulus selectivity of visual cortical cells in senescent rhesus monkeys. Nat Neurosci 2000, 3:384-390.

9. Leventhal AG, Wang Y, Pu M, Zhou Y, Ma Y: GABA and Its Agonists Improved Visual Cortical Function in Senescent Monkeys. Science 2003, 300:812-815.

10. Yang Y, Liang Z, Li G, Wang Y, Zhou Y, Leventhal AG: Aging affects contrast response functions and adaptation of middle temporal visual area neurons in rhesus monkeys. Neuroscience 2008, 156:748-757.

11. Kamal B, Holman C, de Villers-Sidani E: Shaping the aging brain: role of auditory input patterns in the emergence of auditory cortical impairments. Front Syst Neurosci 2013, 7:52.

12. David-Jürgens M, Churs L, Berkefeld T, Zepka RF, Dinse HR: Differential Effects of Aging on Foreand Hindpaw Maps of Rat Somatosensory Cortex. PLOS ONE 2008, 3:1-11.

13. Hua T, Kao C, Sun $Q$, Li X, Zhou Y: Decreased proportion of GABA neurons accompanies age-related degradation of neuronal function in cat striate cortex. Brain Res Bull 2008, 75:119-125.

14. Ding $Y$, Zheng $Y$, Liu $T$, Chen $T$, Wang $C$, Sun $Q$, Hua $M$, Hua $T$ : Changes in GABAergic markers accompany degradation of neuronal function in the primary visual cortex of senescent rats. Sci Rep 2017, 7:14897. 
15. Lalwani P, Gagnon H, Cassady K, Simmonite M, Peltier S, Seidler RD, Taylor SF, Weissman DH, Polk TA: Neural distinctiveness declines with age in auditory cortex and is associated with auditory GABA levels. Neurolmage 2019, 201:116033.

16. Cassady K, Gagnon H, Lalwani P, Simmonite M, Foerster B, Park D, Peltier SJ, Petrou M, Taylor SF, Weissman $\mathrm{DH}$, et al.: Sensorimotor network segregation declines with age and is linked to GABA and to sensorimotor performance. Neurolmage 2019, 186:234-244.

17. Sommer VR, Fandakova Y, Grandy TH, Shing YL, Werkle-Bergner M, Sander MC: Neural pattern similarity differentially relates to memory performance in younger and older adults. $J$ Neurosci 2019, doi:10.1523/jneurosci.0197-19.2019.

18. St-Laurent M, Abdi H, Bondad A, Buchsbaum BR: Memory Reactivation in Healthy Aging: Evidence of Stimulus-Specific Dedifferentiation. J Neurosci 2014, 34:4175-4186.

19. St-Laurent M, Buchsbaum BR: How Multiple Retrievals Affect Neural Reactivation in Young and Older Adults. J Gerontol Ser B 2019, doi:10.1093/geronb/gbz075.

20. Zheng L, Gao Z, Xiao X, Ye Z, Chen C, Xue G: Reduced Fidelity of Neural Representation Underlies Episodic Memory Decline in Normal Aging. Cereb Cortex 2018, 28:2283-2296.

21. Trelle AN, Henson RN, Simons JS: Neural evidence for age-related differences in representational quality and strategic retrieval processes. Neurobiol Aging 2019, doi:10.1016/j.neurobiolaging.2019.07.012.

22. Barron HC, Garvert MM, Behrens TEJ: Repetition suppression: a means to index neural representations using BOLD? Philos Trans R Soc B Biol Sci 2016, 371:20150355.

23. Goh JO, Suzuki A, Park DC: Reduced neural selectivity increases fMRI adaptation with age during face discrimination. Neurolmage 2010, 51:336-344.

24. Reagh ZM, Noche JA, Tustison NJ, Delisle D, Murray EA, Yassa MA: Functional Imbalance of Anterolateral Entorhinal Cortex and Hippocampal Dentate/CA3 Underlies Age-Related Object Pattern Separation Deficits. Neuron 2018, 97:1187-1198.e4.

25. Yassa MA, Mattfeld AT, Stark SM, Stark CEL: Age-related memory deficits linked to circuit-specific disruptions in the hippocampus. Proc Natl Acad Sci 2011, 108:8873-8878.

26. Bowman CR, Chamberlain JD, Dennis NA: Sensory representations supporting memory specificity: Age effects on behavioral and neural discriminability. J Neurosci 2019, 39:2265-2275. Examines item-level neural differentiation in young and older adults during memory retrieval with multivoxel classifiers trained to discriminate between exact repetitions of studied objects (targets) and two different classes of similar objects.

27. Park DC, Polk TA, Park R, Minear M, Savage A, Smith MR: Aging reduces neural specialization in ventral visual cortex. Proc Natl Acad Sci 2004, 101:13091-13095. 
28. Voss MW, Erickson KI, Chaddock L, Prakash RS, Colcombe SJ, Morris KS, Doerksen S, Hu L, McAuley $\mathrm{E}$, Kramer AF: Dedifferentiation in the visual cortex: An $\mathrm{fMRI}$ investigation of individual differences in older adults. Brain Res 2008, 1244:121-131.

29. -Koen JD, Hauck N, Rugg MD: The relationship between age, neural differentiation, and memory performance. J Neurosci 2019, 39:149-162. Examined neural dedifferentiation for scenes and objects during memory encoding. Describes convergent findings for univariate and multivariate measures of neural dedifferentiation for scenes but not objects, and an age-invariant correlation between neural differentiation and multiple measures of cognition.

30. Kanwisher N, Dilks DD: The functional organization of the ventral visual pathway in humans. In The new visual neurosciences. Edited by Werner JS, Chalupa LM. Massachusetts Institute of Technology; 2014:733-748.

31. Carp J, Park J, Hebrank A, Park DC, Polk TA: Age-Related Neural Dedifferentiation in the Motor System. PLOS ONE 2011, 6:e29411.

32. Du Y, Buchsbaum BR, Grady CL, Alain C: Increased activity in frontal motor cortex compensates impaired speech perception in older adults. Nat Commun 2016, 7:12241.

33. Park J, Carp J, Kennedy KM, Rodrigue KM, Bischof GN, Huang C-M, Rieck JR, Polk TA, Park DC: Neural Broadening or Neural Attenuation? Investigating Age-Related Dedifferentiation in the Face Network in a Large Lifespan Sample. J Neurosci 2012, 32:2154-2158.

34. Payer D, Marshuetz C, Sutton B, Hebrank A, Welsh RC, Park DC: Decreased neural specialization in old adults on a working memory task. NeuroReport 2006, 17:487-491.

35. Berron D, Neumann K, Maass A, Schütze H, Fliessbach K, Kiven V, Jessen F, Sauvage M, Kumaran D, Düzel E: Age-related functional changes in domain-specific medial temporal lobe pathways. Neurobiol Aging 2018, 65:86-97.

36. Wang TH, Johnson JD, de Chastelaine M, Donley BE, Rugg MD: The Effects of Age on the Neural Correlates of Recollection Success, Recollection-Related Cortical Reinstatement, and PostRetrieval Monitoring. Cereb Cortex 2016, 26:1698-1714.

37. Thakral PP, Wang TH, Rugg MD: Effects of age on across-participant variability of cortical reinstatement effects. Neurolmage 2019, 191:162-175.

38. Chee MWL, Goh JOS, Venkatraman V, Tan JC, Gutchess A, Sutton B, Hebrank A, Leshikar E, Park D: Age-related Changes in Object Processing and Contextual Binding Revealed Using fMR Adaptation. J Cogn Neurosci 2006, 18:495-507.

39. Barense MD, Henson RNA, Lee ACH, Graham KS: Medial temporal lobe activity during complex discrimination of faces, objects, and scenes: Effects of viewpoint. Hippocampus 2010, 20:389401.

40. Wig GS: Segregated Systems of Human Brain Networks. Trends Cogn Sci 2017, 21:981-996. 
41. Damoiseaux JS: Effects of aging on functional and structural brain connectivity. Neurolmage 2017, 160:32-40.

42. Chan MY, Park DC, Savalia NK, Petersen SE, Wig GS: Decreased segregation of brain systems across the healthy adult lifespan. Proc Natl Acad Sci 2014, 111:E4997-E5006.

43. Varangis E, Habeck CG, Razlighi QR, Stern Y: The Effect of Aging on Resting State Connectivity of Predefined Networks in the Brain. Front Aging Neurosci 2019, 11.

44. Han L, Savalia NK, Chan MY, Agres PF, Nair AS, Wig GS: Functional Parcellation of the Cerebral Cortex Across the Human Adult Lifespan. Cereb Cortex 2018, 28:4403-4423. Reported findings showing that prior evidence of age-related network desegregation in a large lifespan sample (aged 20-89 years) replicate when age-specific sets of network nodes are employed.

45. Cao M, Wang J-H, Dai Z-J, Cao X-Y, Jiang L-L, Fan F-M, Song X-W, Xia M-R, Shu N, Dong Q, et al.: Topological organization of the human brain functional connectome across the lifespan. Dev Cogn Neurosci 2014, 7:76-93.

46. Betzel RF, Byrge L, He Y, Goñi J, Zuo X-N, Sporns O: Changes in structural and functional connectivity among resting-state networks across the human lifespan. Neurolmage 2014, 102:345-357.

47. Song J, Birn RM, Boly M, Meier TB, Nair VA, Meyerand ME, Prabhakaran V: Age-Related Reorganizational Changes in Modularity and Functional Connectivity of Human Brain Networks. Brain Connect 2014, 4:662-676.

48. King BR, van Ruitenbeek P, Leunissen I, Cuypers K, Heise K-F, Santos Monteiro T, Hermans L, Levin O, Albouy G, Mantini D, et al.: Age-Related Declines in Motor Performance are Associated With Decreased Segregation of Large-Scale Resting State Brain Networks. Cereb Cortex 2018, 28:43904402.

49. Iordan AD, Cooke KA, Moored KD, Katz B, Buschkuehl M, Jaeggi SM, Jonides J, Peltier SJ, Polk TA, Reuter-Lorenz PA: Aging and Network Properties: Stability Over Time and Links with Learning during Working Memory Training. Front Aging Neurosci 2018, 9:419.

50. Geerligs L, Renken RJ, Saliasi E, Maurits NM, Lorist MM: A Brain-Wide Study of Age-Related Changes in Functional Connectivity. Cereb Cortex 2015, 25:1987-1999.

51. Chong JSX, Ng KK, Tandi J, Wang C, Poh J-H, Lo JC, Chee MWL, Zhou JH: Longitudinal Changes in the Cerebral Cortex Functional Organization of Healthy Elderly. J Neurosci 2019, 39:5534-5550. Reports evidence for within-individual longitudinal decreases in network segregation, particularly in associative networks involved in higher-order cognition, over 2-3 sessions occurring over intervals ranging between 18 to 24 months.

52. Chan MY, Alhazmi FH, Park DC, Savalia NK, Wig GS: Resting-State Network Topology Differentiates Task Signals across the Adult Life Span. J Neurosci 2017, 37:2734-2745. Demonstrates in a large lifespan sample (aged 20-89 years) that age-related network desegregation is accompanied by reductions in the regional specificity of task-related neural activation for nodes that have high connectivity within their network and low connectivity with other networks. 
53. Park J, Carp J, Hebrank A, Park DC, Polk TA: Neural Specificity Predicts Fluid Processing Ability in Older Adults. J Neurosci 2010, 30:9253-9259.

54. Rugg MD: Interpreting age-related differences in memory-related neural activity. In Cognitive Neuroscience of Aging: Linking Cognitive and Cerebral Aging. Edited by Cabeza R, Nyberg L, Park DC. Oxford University Press; 2017:183-206.

55. Lu H, Liu P: MRI measures on aging: Methodological issues. In Cognitive Neuroscience of Aging: Linking Cognitive and Cerebral Aging. Edited by Cabeza R, Nyberg L, Park DC. Oxford University Press; 2017:9-34.

56. Abdulrahman $\mathrm{H}$, Henson RN: Effect of trial-to-trial variability on optimal event-related fMRI design: Implications for Beta-series correlation and multi-voxel pattern analysis. Neurolmage $2016,125: 756-766$. Evaluates the role of dopamine as a possible mediator of neural dedifferentiation using pharmacological interventions.

57. Dennis NA, Cabeza R: Age-related dedifferentiation of learning systems: an fMRI study of implicit and explicit learning. Neurobiol Aging 2011, 32:2318.e17-2318.e30.

58. St-Laurent M, Abdi H, Burianová H, Grady CL: Influence of Aging on the Neural Correlates of Autobiographical, Episodic, and Semantic Memory Retrieval. J Cogn Neurosci 2011, 23:41504163.

59. Martins B, Ponzio A, Velasco R, Kaplan J, Mather M: Dedifferentiation of emotion regulation strategies in the aging brain. Soc Cogn Affect Neurosci 2015, 10:840-847. 

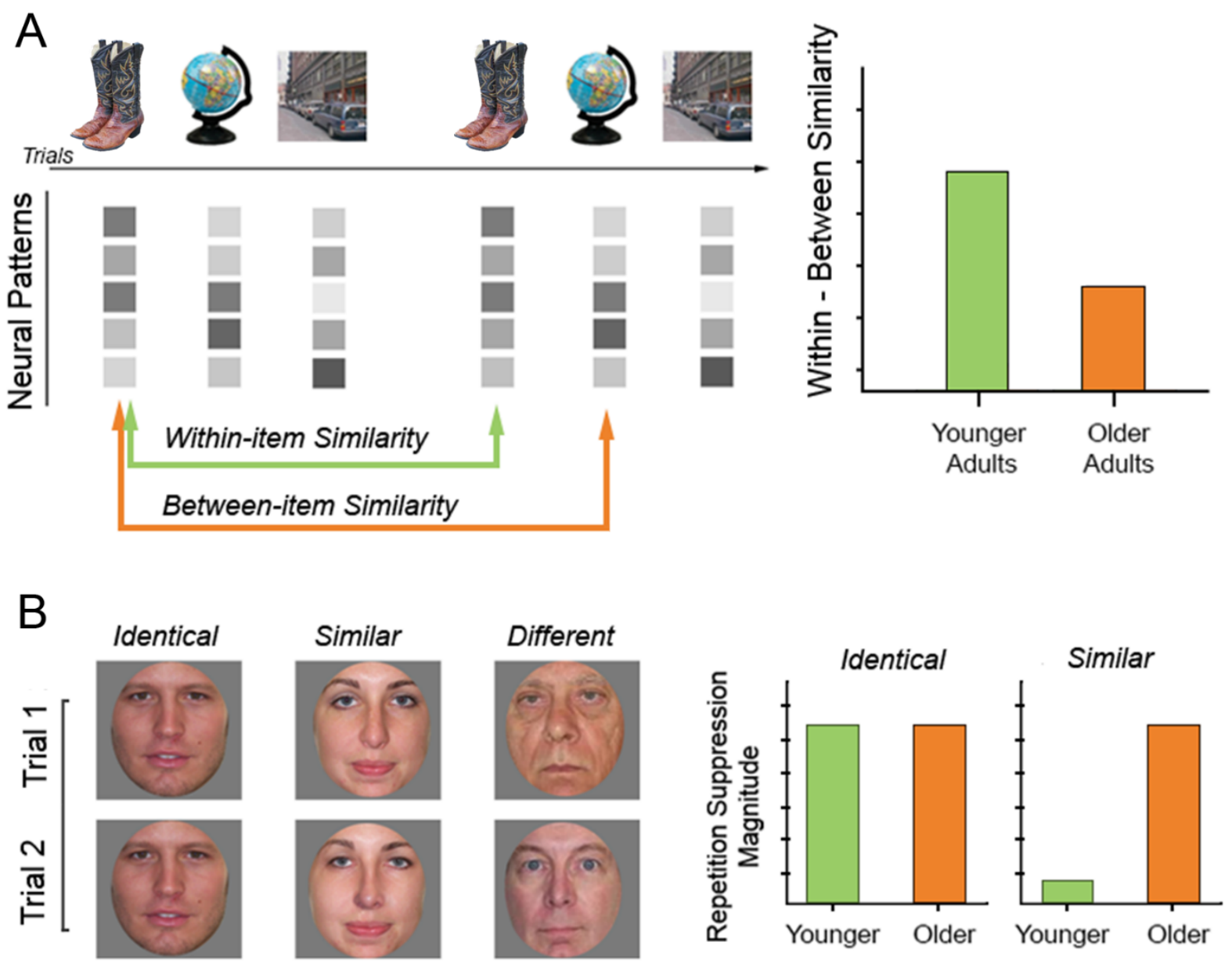

Figure 1. (A) Item-level differentiation can be measured by contrasting the similarity of neural patterns between repetitions of an identical exemplar (within-item similarity) and repetitions of different exemplars of the same category (between-item similarity). As illustrated, age-related dedifferentiation takes the form of smaller within-between similarity differences. (B) Item-level differentiation can also be operationalized by the comparison of 'repetition suppression' effects elicited by repetition of an identical stimulus with the effects elicited by the presentation of a different, but highly similar, stimulus. The logic of this approach is that suppression effects for highly similar foils reflect the re-engagement of overlapping neural representations. Thus, suppression for similar items is thought to be indicative of a low-fidelity neural representation of the first-presented item. 

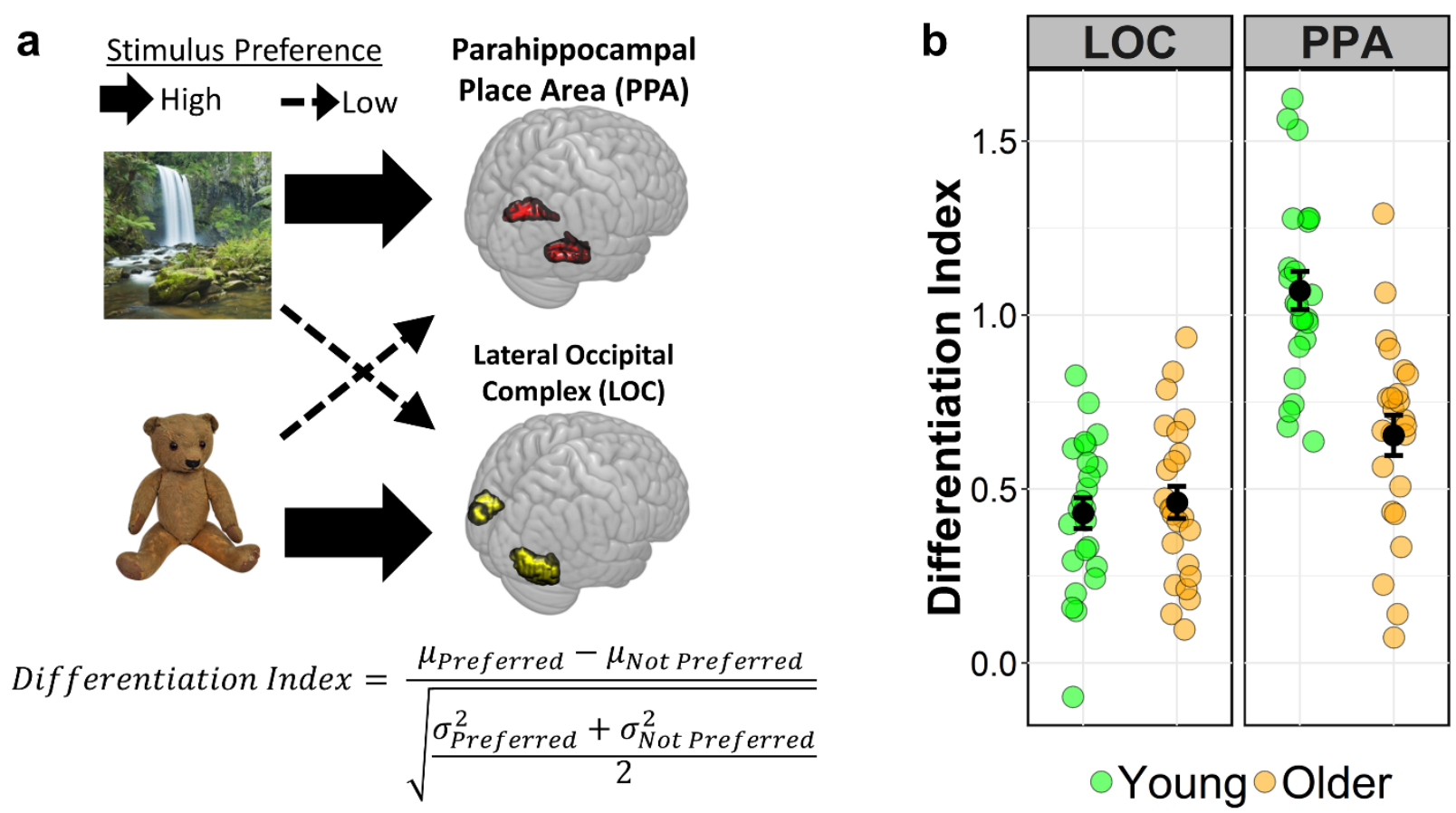

Figure 2. (A) Example of category selective age-related dedifferentiation operationalized as reduced neural selectivity between a region's preferred and not preferred stimulus categories measured with a differentiation index [28], which is one of many ways to quantify category-level differentiation (cf. [7]). (B) Results from Koen et al. [29] illustrating age-related neural dedifferentiation for scenes in the parahippocampal place area, but not for objects in the lateral occipital complex. 

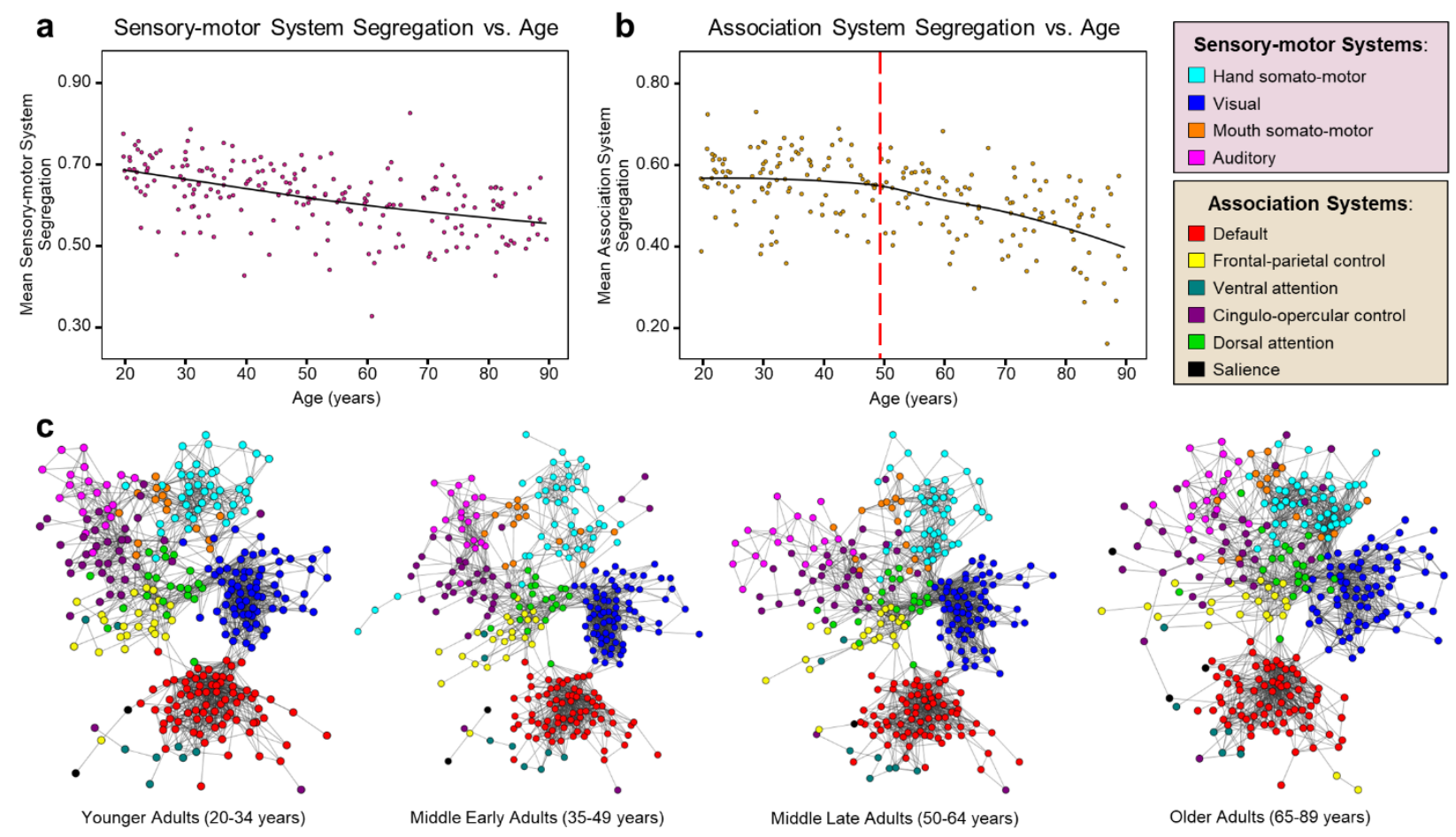

Figure 3. Results from Chan and colleagues [42] depicting age-related neural dedifferentiation at the network level. (A) Network segregation - reflecting the degree to which network nodes show preferential correlations to other nodes within their own functional systems versus nodes in other functional systems - of sensory-motor systems demonstrated a linear decrease with chronological age.

(B) Segregation of association systems showed a nonlinear, positively accelerating relationship with age.

(C) Spring plots of 10 different resting-state networks demonstrating that network desegregation is driven both by decreased within-network connectivity (distance between same-colored nodes) and increased between-network connectivity (increased overlap between differently-colored nodes). 